\title{
A Proposed Currency System for Academic Peer Review Payments Using the BlockChain Technology
}

\author{
Michael Spearpoint ${ }^{1,2}$ \\ 1 Department of Civil and Natural Resources Engineering, University of Canterbury, \\ Christchurch, 8020, New Zealand; michael.spearpoint@canterbury.ac.nz \\ 2 Olsson Fire \& Risk, Manchester M4 6WX, UK \\ Academic Editor: Robert Campbell \\ Received: 23 May 2017; Accepted: 10 July 2017; Published: 14 July 2017
}

\begin{abstract}
Peer review of scholarly papers is seen to be a critical step in the publication of high quality outputs in reputable journals. However, it appears that there are few incentives for researchers to agree to conduct suitable reviews in a timely fashion and in some cases unscrupulous practices are occurring as part of the production of academic research output. Innovations in internet-based technologies mean that there are ways in which some of the challenges can be addressed. In particular, this paper proposes a new currency system using the BlockChain as its basis that provides a number of solutions. Potential benefits and problems of using the technology are discussed in the paper and these will need further investigation should the idea develop further. Ultimately, the currency could be used as an alternative publication metric for authors, institutions and journals.
\end{abstract}

Keywords: peer review; BlockChain; currency

\section{Introduction}

Almost every research academic is aware of the current environment in which both institutions and individuals are subject to some form of assessment and a considerable proportion of the scoring is often weighted on the production of scholarly outputs, typically as papers in reputable journals. Apart from the obvious authors and readers, these journals typically have a small number of editors who do most of the day-to-day management of the intellectual material; there might also be a larger editorial pool from the discipline who oversee the journal, and then there are those people who take on the role of reviewers which could include all of the previously listed groups of people. The production of the journal will also include a publisher that will include those that publish for profit and those that publish through a non-profit entity such as a learned society. Irrespective of the details of the process, the production of an academic journal is an economic system and like any such system the publishing process presents a number of intended and unintended incentives.

Due to the anonymous nature of peer review, there appears to be a lesser incentive for people to carry out this part of the publication process when compared to authoring articles or being part of the editorial activity. Although some journals will list those that have carried out reviews on its behalf, it is more difficult to provide a measure of the number, quality and timeliness of those reviews. This paper considers how it might be possible to provide a greater incentive to carry out peer review and to raise the quality of those reviews using some of the currently available internet-based technologies. The proposal also provides a mechanism to track the review process and has the potential for wider application within the academic publishing environment as an alternative metric to those used at present. 


\section{Peer Review}

One of the primary expectations is that the publication of a journal paper occurs after the article has undergone a peer review. Research is already available in the literature [1,2] that acknowledges there are numerous limitations with this system including the fact that the review process can be slow and cumbersome. The review process also exhibits various forms of bias, including a sometimes arbitrary outcome as to whether a paper gets rejected or not [1]. From an unwanted incentive perspective, obtaining peer reviews also potentially presents conflicts of interest where a reviewer might deliberately reject a paper from a competing institution given the growing intensity in ranking assessments.

In order to expedite the peer review process, some journals allow the authors to submit a list of suggested reviewers. However, it is conceivable that names are nominated from a small group of well-known individuals within the discipline who subsequently become overloaded with requests; alternatively, nominations may be of people who, from the editors' perspective, are insufficiently qualified individuals; reviewers may be nominated from the same institution as the author/s; reviewers may have already agreed with the authors to give a positive review; they may be fake reviewers where an author (or an agent on their behalf) has set up a separate, alternative account in a different name and effectively reviewed themselves [3]. The use of nominated reviewers is somewhat mitigated where an editor might choose several reviewers of which only one, say, is from the nominated list. Some journals require that authors must only submit institutional email addresses for recommended reviewers they nominate [4]. However, this author has been made aware that there are now journals that automatically prevent an editor from selecting one of the recommended reviewers due to conflict of interest and require that editors provide justification if they do want to pick a recommended reviewer.

From a personal point of view, the author has taken on all the roles listed in the Introduction. The author has been on the receiving end of very slow reviews of up to nearly two years from submission, hugely conflicting reviews, reviews that are not sufficiently constructive to improve a submission, etc. Similarly, as a past associate editor of a journal, the author knows how difficult it is to find reviewers, to get reviewers to make timely submissions, to obtain unbiased reviews that are of sufficient quality and to identify where various types of unscrupulous activities are taking place. In many cases, highly regarded peer reviewers are likely to be busy already reviewing papers as well as carrying out all of the other duties expected of them which if nothing else includes trying to maintain their own publication record. However, there was the impression by this author that some individuals would never agree to doing peer reviews even for journals they were publishing in and it seems reasonable to expect those people to contribute to their discipline by taking on the role of a peer reviewer once they have published a certain body of work.

For all of the identified problems with peer review, it seems the process will be staying around for the foreseeable future since it provides a mechanism to maintain some form of quality control within a discipline. However, with the advent of the internet and all of the associated innovations there is scope to make significant changes. Some of these changes are already evident with the advent of open access publishing, the association of DOIs to scholarly outputs, readily available repository sites such as ResearchGate (researchgate.net), the non-proprietary ORCID (Open Researcher and Contributor ID) author identification system (orcid.org), the Publons peer review platform (publons.com) etc. Even the process of peer review could change, for example, Adler [1] discusses a 'crowd-sourcing' approach to peer review. In the paper by Wood [5], he proposed a number of suggestions to modify the publication and review system. However, Wood did not discuss the direct incentives to carry out quality reviews in a timely fashion.

\section{Proposed Currency System}

\subsection{BlockChain Technology}

One incentive to improve the peer review process might be to pay the reviewers with 'real' money but that has potential conflicts of interest. Instead, the internet presents new technologies 
that allow for alternative currency exchange mechanisms using the BlockChain [6]. The advantages of the BlockChain as a means of exchange are discussed at length in the literature but essentially it is a distributed accounting system that is not owned by any specific organisation, it is difficult to corrupt and transactions are anonymous. In this alternative proposed exchange system, reviewers are paid with a cryptocurrency similar to bitcoin (here referred to as r-coin for 'ReviewCoin' or maybe 'ResearchCoin') for each peer review they undertake.

In order for this exchange system to work, authors will need to register an ID using a system such as ORCID and then submit their ID to the journal or through Publons when doing a peer review. The r-coin is only paid once an editor accepts the review in terms of meeting the journal's quality requirements. The payment of the amount of r-coin could also be related to timeliness where less $r$-coin is paid if a review is late. Once earned, these r-coins are then used to 'pay' for the cost of publishing in a journal and since the system is completely open, it could be any other journal, not necessarily the one that carried out the review or even the same publisher.

In order for the system to work, there would also need to be a registration process for new journals to join the r-coin currency to prevent unscrupulous people from setting up publications that do not conform to the rules agreed by the community. Journals that are produced by established publishers would not be an issue but there are already a large number of predatory journals, with their associated implications [7], which should be excluded from participating. However, there would need to be a body that decides which journals were acceptable. The body could consist of those publishers already in the system but they may have an incentive to prevent other publishers from taking part. Lists such the Directory of Open Access Journals (doaj.org) provide one means to identify reputable open access journals but such lists are not perfect. Ultimately, there needs to be a community solution to this issue as exemplified by Clark and Smith [7].

Finally, the r-coin system does not negate the need for publishers to obtain 'real' money to pay for servers to host their products, staff to manage the publication process etc. This is no different to the present and so there will still be a need for submission and/or subscription fees such that the r-coin system only provides a 'right to publish' mechanism. If exchange with other currencies was enabled, then the need for 'real' money could diminish; however, allowing such exchange could lead to other undesirable knock-on effects which are briefly discussed later in this paper.

\subsection{Transaction Mechanisms}

Clearly, if there is a cost to submit papers to journals, then an author will need an r-coin budget. A new researcher, such as a PhD student who is a co-author on their first paper, may not have yet reached a stage in which they are experienced enough in their discipline to enable them to carry out peer reviews in order to earn $r$-coin. Therefore, it is proposed that an author would be given a certain amount of r-coin during the ID registration process. This means they will then have an initial $r$-coin budget so that they can then start submitting papers to journals.

Once the currency is operating, there will need to be a decision made on various transaction costs such as determining how much a person should be paid for reviewing for a paper. Similarly, the cost of submitting a paper to a journal would need to be agreed and whether that cost was the same for all journals or journals could set their own price. When multiple authors are involved in submitting a paper, then should the cost be shared equally, or only the corresponding author pay or the authors could decide their own r-coin contribution that could be a reflection of their contribution to the paper. At this stage, this author does not have particular recommendations on these transaction costs.

Another matter that needs to be considered is whether the r-coins should remain in perpetuity or should they decay over time. Having the number decay would provide an incentive for reviewers to keep on contributing to the process if they wanted to carry on publishing papers. Thus, unlike with the bitcoin currency in which the pool of coins becomes increasingly more difficult to increase, the r-coin pool expands when people register and when they complete a review. The pool shrinks once 
$\mathrm{r}$-coins are returned to a publisher to be effectively withdrawn from circulation and/or r-coins decay through lack of use.

As with any economic system, there will be unintended outcomes so it is worthwhile examining what those outcomes might be and how they might be addressed. Requiring authors and reviewers submit their ID eliminates the likelihood of self-review if the same ID is used for both transactions if the appropriate check is made. However, one potential outcome of the r-coin system is that unscrupulous authors might consider registering several IDs to increase their r-coin budget and open the possibility of self-review. By having reviewers associate their ID through the ORCID system, it would mean that a person that had multiple IDs would then have to distribute their scholarly outputs across those IDs, making it difficult to demonstrate a coherent body of work if their ORCID profile was used to assess their contributions. However, there would be an even greater incentive for the person to only have a single ID if they were paid more r-coins per review the more reviews that were completed.

\subsection{Expansion of the R-Coin Exchange}

The r-coin exchange system has a number of potential areas in which it could be expanded beyond the peer review process. If Wood's [1] model of open peer review were adopted, then this could be another mechanism to earn r-coins, albeit the exchange rate could be a lot less than that on an invited review from a journal. A requirement for the reviewer to submit their ID and having such reviews to get some form of mark of acceptance, maybe from the journal editorial board before a payment was made, would reduce instances of a fully anonymous review that could simply be deliberately abusive or misinformed. The availability of open reviews would also allow for and maybe encourage reviews of reviews with an associated r-coin payment rate.

The r-coin system could be expanded to include paying authors once papers had been published with the potential for a non-linear payment system so that authors earn more per paper as they publish more papers. Similarly, the r-coin earned could be proportioned to the r-coin payment contribution when the paper was originally submitted.

Once a paper is published, then it would be possible for the author to earn r-coins for citations. In particular, it might be possible that r-coins are only earned for those citations in which the author of the citing paper decides the original paper has sufficient merit. This author is aware of one journal where citing authors already nominate papers which they consider to be noteworthy in which comments are given against selected cited publications in the reference list, for example, see reference [8].

The r-coin system could be expanded to allow owners to pay for journal subscriptions as an extension of the practice of awarding limited access to a publisher's collection as part of the recognition to reviewers. This might be useful for, say, retired individuals who have left their parent institution, who have no need to publish but still want to maintain the ability to read particular journals.

With the potential application of the r-coin system to reviews and publications, it would also be possible to track by what means the coins were earned, whether through an invited review, by open review as well as by tracking a journal through its ISSN reference. This would also allow for automatic recognition of peer review activity, such as the journal and year of review, through ORCID if that were used as the ID. The eventual outcome of the r-coin system would mean that there was also a mechanism to rate individual researchers and to rate institutions by combining the earnings of individuals over whatever period is appropriate.

\subsection{Inter-Currency Exchange}

Should the r-coin system become established, then owners could potentially exchange them amongst themselves so that someone with excess $r$-coin could give them to another who has an insufficient amount. A possible scenario could be where a principal investigator (PI) asks everybody in their lab, including research associates and/or lab technicians, who are not planning on authoring 
papers to sign up for an ORCID and then transfer their r-coin to the PI. This would compromise the intention of the r-coin system and thus suggests that inter-author exchange be prohibited.

Following on from the above, the issue of whether r-coin could be exchanged for bitcoin or another currency would be possible and desirable. It is not the intention of the proposal to suggest that such transactions would be appropriate but with any emerging technology it can be difficult to know how things might develop. At this stage, it appears that it would probably be much more difficult to go from another currency to r-coin as the sale of r-coin would be restricted to the journals, so long as those journals acted scrupulously. However, it might not be so easy to prevent transactions from r-coin to another currency although this would appear not to be something that would fundamentally impact the intention of the r-coin system in the context of the peer review and publication process, unless this became a means to facilitate a 'real' money shadow payment mechanism for reviews.

Such possibilities need to be addressed but transactions should be able to be tracked through the BlockChain which would allow them to be blocked at the time or reconciled at a later date.

\section{Conclusions}

This paper proposes a new exchange system for the peer review procedure that could provide a number of improvements to the process. The exchange system would adopt various internet-based technologies that specifically includes a currency that uses the underlying BlockChain technology. The r-coin proposal does not solve every problem associated with the current academic publishing environment but does provide mechanisms to mitigate some of the challenges associated with the peer review process with the potential for further expansion should it be widely adopted.

Obviously, the system would need journals to agree to participate in the proposal. Much of the technology already exists including the BlockChain, DOIs and ORCID and if citations were to be included, then a system such as Google Scholar is available. In the end, the main incentive is that a journal and its editors need high quality peer reviews so as to maintain the standing of the journal and therefore attract top authors that will in turn lead to a strong citation pedigree.

Since the author of this paper is not an expert in many details of the technology, this proposed currency system may have, as yet, hidden unintended consequences. It is likely that there will be many others who have much deeper insights into what can or cannot be implemented with the technology, so the author encourages readers to discuss the suggestion and point out improvements.

Acknowledgments: The author would like to thank Richard Hull, University of Central Lancashire, Bart Merci, Ghent University, Guillermo Rein, Imperial College London and Alexander Morgan, University of Dayton for their useful discussion related to the ideas presented in this paper.

Conflicts of Interest: The author declares no conflict of interest in the preparation of this paper.

\section{References}

1. Adler, J.R. A new age of peer reviewed scientific journals. Surg. Neurol. Int. 2012, 3, 145. [CrossRef] [PubMed]

2. Laframboise, D. Peer Review-Why Scepticism is Essential; GWPF Report 20; The Global Warming Policy Foundation: London, UK, 2016; 40p ${ }^{1}$.

3. Moylan, E. Inappropriate Manipulation of Peer Review. BioMed Central Blog. Available online: https://blogs. biomedcentral.com/bmcblog/2015/03/26/manipulation-peer-review/ (accessed on 7 July 2017).

4. Cury, A. 5 Tips to Help Prevent Reviewer Fraud. Available online: https://hub.wiley.com/community/ exchanges/discover/blog/2017/05/01/5-tips-to-help-prevent-reviewer-fraud (accessed on 7 July 2017).

5. Wood, M. Journals, Repositories, Peer Review, Non-Peer Review, and the Future of Scholarly Communication. 2013. Available online: https:/ / papers.ssrn.com/sol3/papers.cfm?abstract_id=2356633 (accessed on 7 July 2017).

1 By citing this reference the author is not necessarily subscribing to the implications on climate change research but the reference does provide some valid points regarding the limitations of peer review in general. 
6. Crosby, M.; Pattanayak, P.; Verma, S.; Kalyanaraman, V. BlockChain technology: Beyond Bitcoin. Appl. Innov. Rev. 2016, 2, 6-10.

7. Clark, J.; Smith, R. Firm action needed on predatory journals. BMJ 2015, 350. [CrossRef] [PubMed]

8. Marchand, A.; Haddad, S. Simultaneous exposures to heat and chemicals and the impact on toxicokinetics and biomonitoring. Curr. Opin. Toxicol. 2017, 4, 22-27. [CrossRef] 